\title{
DIRECT THERMOMAGNETIC SPLITTING OF WATER
}

\author{
R. L. CURL \\ Department of Chemical Engineering, The University of Michigan, Ann Arbor, MI 48109, U.S.A. \\ (Received for publication 26 July 1978)
}

\begin{abstract}
The application of a magnetic field to water tends to cause its decomposition into hydrogen and oxygen. Based upon the thermomagnetochemistry of the phenomenon. a process is suggested for carrying out the reaction and separating the product hydrogen and oxygen. The process would have nearly Carnot efficiency, although the requisite magnetic field $\left(\sim 10^{4}\right.$ tesla) is not at present attainable.
\end{abstract}

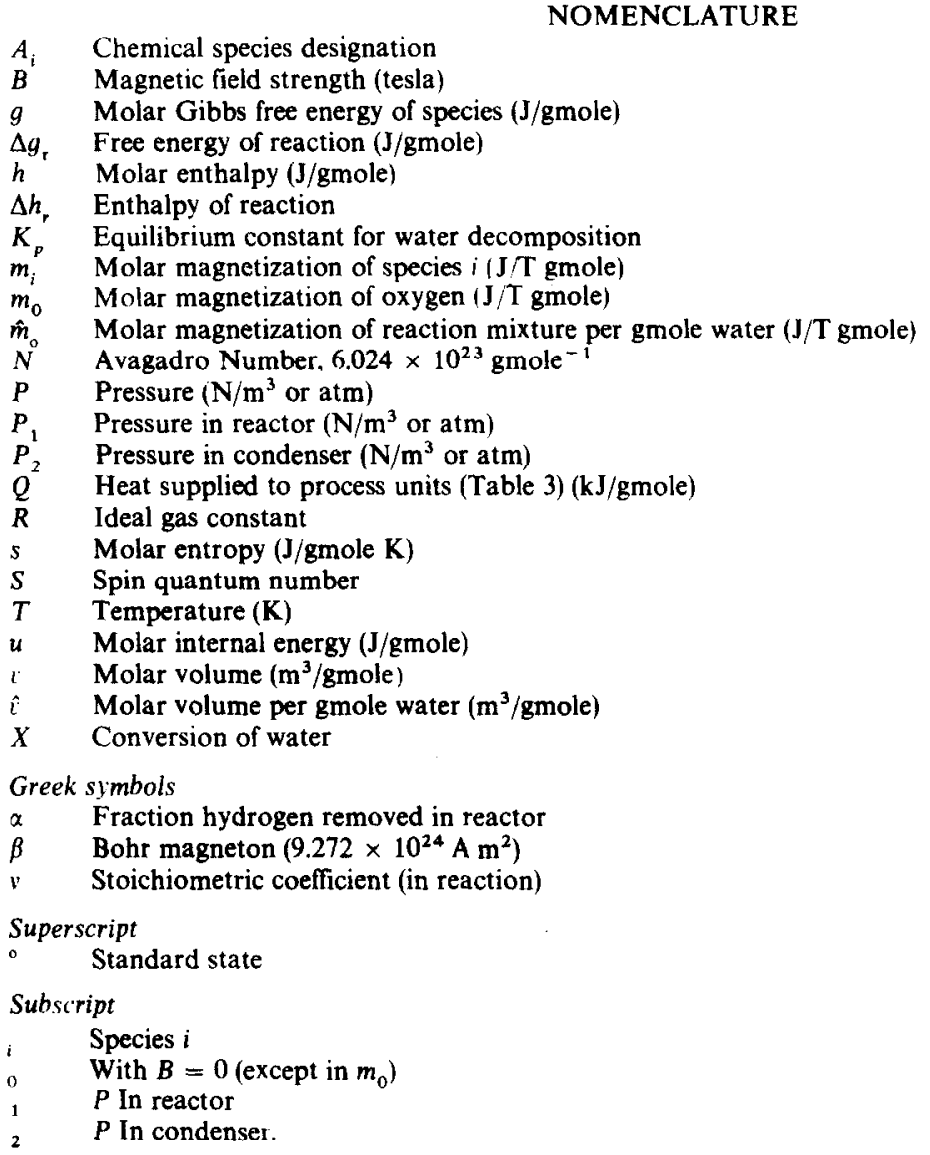

\section{INTRODUCTION}

WATER is too stable to be easily thermally decomposed at moderate temperatures [1]. It is, however. well known that molecular oxygen is a paramagnetic substance and is therefore attracted into a magnetic field. Since water and hydrogen are not paramagnetic, the application of a magnetic field to water should tend to "extract" the oxygen and enhance the reaction

$$
\mathrm{H}_{2} \mathrm{O}=\mathrm{H}_{2}+\frac{1}{2} \mathrm{O}_{2} \text {. }
$$

Previous studies by Delhez [2,3] and Chen et al. [4] have shown that the shift in the equilibrium of a chemical reaction involving paramagnetic substances is quite small with "ordinary" magnetic 
fields of the order of 1 tesla ( $1 \mathrm{~T}=10^{4} \mathrm{G}$ ). It is the purpose here to explore the magnetic fields required for a practical shift in the equilibrium of reaction (1) and to suggest a system by which water might be thermomagnetically decomposed into hydrogen and oxygen.

\section{THERMOMAGNETOCHEMISTRY}

The following is a simplified and idealized thermodynamic analysis of chemical systems with a magnetic field.

It is known (e.g. Guggenheim [5]; Hsieh [6]) that the first-law expression including magnetization is, on a molar basis,

$$
\mathrm{d} u=T \mathrm{~d} s+B \mathrm{~d} m-P \mathrm{~d} v,
$$

where $u=$ molar internal energy $(\mathrm{J} /$ gmole $)$.

$T=$ Temperature $(\mathbf{K})$.

$s$ = molar entropy $(\mathrm{J} / \mathrm{K} \mathrm{kgmole})$,

$B=$ magnetic field strength $\left(\mathrm{T}\left(=\mathrm{Wb} / \mathrm{m}^{2}=\mathrm{J} / \mathrm{A} \mathrm{m}^{2}\right)\right)$,

$m=$ molar magnetization $\left(\mathrm{Am}^{2} /\right.$ gmole $(=\mathrm{J} / \mathrm{T}$ gmole $)$,

$P=$ pressure $\left(\mathrm{N} / \mathrm{m}^{2}\right)$ and

$v=$ molar volume $\left(\mathrm{m}^{3}\right)$.

The magnetic Gibbs free energy is defined as $[5,6]$

from which it follows that

$$
g=u+P v-B m-T s \text {. }
$$

$$
\mathrm{d} g=-s \mathrm{~d} T-m \mathrm{~d} B+v \mathrm{~d} P .
$$

For an ideal gas mixture at constant temperature, the molar free energy of species " $"$ " may be obtained by integrating (4), with $v_{i}=R T / P_{i}$, to give

$$
g_{i}(B, T)=g_{i 0}-\int_{0}^{B} m_{i} \mathrm{~d} B+R T \ln P_{i}
$$

where $g_{i 0}^{\mathrm{O}}$ is the standard state free energy of " $i$ " at $T$ and $B=0$. For the chemical reaction $\Sigma v_{i} A_{i}=0$ where the $v_{i}$ are the stoichiometric coefficients of the reaction, it follows that, at equilibrium,

$$
\Delta g_{r}=\Sigma v_{i} g_{i 0}-\int_{0}^{B} \Sigma v_{i} m_{i} \mathrm{~d} B+R T \Sigma v_{i} \ln P_{i}=0 .
$$

Since $\Sigma v_{i} g_{i 0}^{0}=\Delta q_{r 0}^{0}$, the standard state free energy of reaction, and $\Sigma v_{i} \ln P_{i}=\ln K_{p}\left(K_{p}=\right.$ pressure equilibrium constant),

$$
-R T \ln K_{p}=\Delta g_{r 0}-\int_{0}^{B} \Sigma v_{i} m_{i} \mathrm{~d} B
$$

For the water splitting reaction (1), only oxygen is significantly paramagnetic while the diamagnetism of hydrogen or water is weak in comparison. Neglecting these, and writing hereafter $m_{\mathrm{O}_{2}}=m_{\mathrm{O}}$. and with $v_{\mathrm{O}_{2}}=\frac{1}{2}$, we obtain

$$
\Delta g_{r}^{\circ}=-R T \ln K_{p}=\Delta g_{r 0}^{\circ}-\frac{1}{2} \int_{0}^{B} m_{0} \mathrm{~d} B .
$$

Magnetization, therefore, reduces the apparent standard state free energy of reaction, increases $K_{p}$. and promotes the decomposition of water.

\section{MOLAR MAGNETIZATION OF MOLECULAR OXYGEN}

Oxygen is a substance with a net electron spin quantum number of $S=1$. narrow multiplet interval and little spin-orbital coupling. Its magnetization is known to be given by the Brillouin function (Van Vleck [7]; Guggenheim [5])

$$
m_{0}=(2 S+1) N \beta \operatorname{ctnh}((2 S+1) N \beta B / R T)-N \beta \operatorname{ctnh}(N \beta B / R T),
$$


where $N=$ Avagadro number $\left(6.024 \times 10^{23}\right.$ gmole $\left.^{-1}\right)$,

$\beta=$ Bohr magneton $\left(9.272 \times 10^{24} \mathrm{~A} \mathrm{~m}^{2}\right)$,

$\left(N_{\beta}=5.586 \mathrm{Am}^{2} / \mathrm{gmole}\right)$ and

$R=8.314 \mathrm{~J} / \mathrm{K}$ gmole.

With the substitution of eq. (9), and integration, eq. (8) becomes, with $S=1$.

$$
\Delta g_{r}^{\circ}=\Delta g_{r 0}-\frac{1}{2} R T \ln \left(\frac{\sinh (3 N \beta B / R T)}{3 \sinh (N \beta B / R T)}\right) .
$$

It follows, by application of the Van't Hoff thermodynamic identity.

$$
\frac{\partial(\Delta g / T)}{\partial(1 / T)}=\Delta h
$$

that the standard-state enthalpy of reaction is given by

$$
\Delta h_{r}^{\circ}=\Delta h_{r 0}^{\circ}-\frac{1}{2} m_{\mathrm{o}} B=\Delta h_{r 0}^{\circ}-\frac{1}{2} N \beta B\left(3 \operatorname{ctnh} \frac{3 N \beta B}{R T}-\operatorname{ctnh} \frac{N \beta B}{R T}\right) .
$$

When $B=0$,

$$
\Delta g_{r 0}^{\circ}=-R T \ln K_{p 0}
$$

and eq. (8) may be expressed as

$$
\ln \frac{K_{p}}{K_{p^{0}}}=\frac{1}{2} \ln \left(\frac{\sinh (3 N \beta B / R T)}{3 \sinh (N \beta \bar{B} / R T)}\right)
$$

For small magnetic fields $(B \rightarrow 0)$, this reduces to

$$
\ln \frac{K_{p}}{K_{p 0}^{\prime}}=\frac{2(N \beta B)^{2}}{3(R T)^{2}}
$$

in agreement with the result obtained by Chen et al. [4]. This result also follows from assuming that the molecular magnetic susceptibility is given by Curie's Law. The Brillouin function was used here because, as will be seen, the magnetic fields required for a significant shift of the equilibrium of re-

\begin{tabular}{|c|c|c|c|c|c|c|c|c|}
\hline \multirow[b]{2}{*}{$\begin{array}{c}B \\
\text { (Tesla) }\end{array}$} & \multicolumn{2}{|c|}{$T=300 \mathrm{~K}$} & \multicolumn{2}{|c|}{$T=600 \mathrm{~K}$} & \multicolumn{2}{|c|}{$T=900 \mathrm{~K}$} & \multicolumn{2}{|c|}{$T=1200 \mathrm{~K}$} \\
\hline & $\begin{array}{c}\Delta h_{r}^{\prime \prime} \\
(\mathbf{k J} / \text { gmole })\end{array}$ & $-\log _{10} K_{p}$ & $\begin{array}{c}\Delta h_{r}^{\circ} \\
\text { (kJ/gmole) }\end{array}$ & $-\log _{10} K_{p}$ & $\begin{array}{c}\Delta h_{r}^{\circ} \\
\text { (kJ/gmole) }\end{array}$ & $-\log _{10} K_{p}$ & $\begin{array}{c}\Delta h \\
(\mathrm{~kJ} / \mathrm{gmole})\end{array}$ & $-\log _{10} K$ \\
\hline 0 & 242 & 39.8198 & 245 & 18.6620 & 247 & 11.5135 & 249 & 7.9003 \\
\hline $10^{3}$ & 236 & 39.0834 & 239 & 18.3901 & 243 & 11.3751 & 246 & 7.8176 \\
\hline $10^{4}$ & 186 & 30.3336 & 189 & 14.0382 & 191 & 8.5105 & 193 & 5.7076 \\
\hline $10^{5}$ & -317 & -57.1888 & -314 & -29.7230 & -311 & -20.6637 & -310 & -16.1730 \\
\hline $4.119 \times 10^{4}$ & 11.7 & 0 & & & & & & \\
\hline $4.329 \times 10^{4}$ & 0 & -2.0399 & & & & & & \\
\hline $3.887 \times 10^{4}$ & & & 27.7 & 0 & & & & \\
\hline $4.382 \times 10^{4}$ & & & 0 & -2.4063 & & & & \\
\hline $3.625 \times 10^{4}$ & & & & & 44.7 & 0 & & \\
\hline $4.425 \times 10^{4}$ & & & & & 0 & -2.5919 & & \\
\hline $3.347 \times 10^{4}$ & & & & & & & 62.1 & 0 \\
\hline $4.458 \times 10^{4}$ & & & & & & & 0 & -2.699 \\
\hline
\end{tabular}
action (1) lead to paramagnetic (electron spin) saturation.

From eqs (11) and (13) we may calculate $\Delta h_{r 0}^{\circ}$ and $-\log _{10} K_{p}$ as a function of $B$ and $T$. Some results are shown in Table 1.

TABLE 1. Heat of reaction and equilibrium constant, for water decomposition, as a function of mag. netic field strength

$\Delta H_{r 0}^{\circ}$ and $-\log _{10} K_{p 0}$ from Ref. [8]. 
It is immediately apparent that very intense magnetic fields, of the order of $10^{4} \mathrm{~T}$, are required to increase significantly the equilibrium constant for water decomposition. High fields are still required at moderately elevated temperatures, even though $\Delta g_{r 0}^{\circ}$ decreases, because the molar magnetization decreases with increasing temperature. The fields required for either $\Delta h_{r}^{\circ}=0$ or $\Delta g_{r}^{\circ}=0\left(K_{p}=1\right)$ are also shown at each temperature. It is interesting that the heat of reaction can be driven to zero but this is not unexpected as the effect of the magnetic field is essentially to reduce the entropy of reaction.

\section{INTENSE MAGNETIC FIELDS}

Paramagnetic saturation occurs for magnetic fields in excess of $10^{4} \mathrm{~T}$. For these large field strengths relations (10) and (11) simplify to

$$
\ln \frac{K_{p}}{K_{p 0}}=\frac{2 N \beta B}{R T}
$$

and

$$
\Delta h_{r}^{\circ}=\Delta h_{r 0}^{\circ}-N \beta B
$$

The practical attainment of steady, non-dissipative, magnetic fields of $10^{4} \mathrm{~T}$ is not possible from current superconducting magnet technology $[10,11]$ because of inherent physical limitations on the superconductivity phenomenon as well as mechanical strength limitations. In addition, an appalling amount of energy is stored in such a field $\left[4 \times 10^{7} \mathrm{~J} / \mathrm{cm}^{3} !\right]$. The highest continuous field available today is of the order of $30 \mathrm{~T}$ [9] and this is by means of a hybrid superconducting-conventional coil arrangement that consumes considerable power. Fields up to $8.5 \mathrm{~T}$ have been generated by nondissipative superconducting magnets $[9,10]$. Up to $10^{3} \mathrm{~T}$ has been generated momentarily (approx. $2 \mu \mathrm{sec}$ ) in the laboratory [11]. Fields of $10^{4} \mathrm{~T}$ are found, however, in certain stellar bodies [12].

In comparison with a direct high temperature $(\sim 2400 \mathrm{~K})$ water-splitting technology [1], a direct thermomagnetic technology appears to be of similar if not greater difficulty. Nevertheless, the nature of the difficulty is very different and, while keeping the above considerations in mind, it appears worthwhile to visualize a water-splitting system based on thermomagnetochemistry. The ultimate benefits to be gained have been amply recorded in the pages of the International Journal of Hydrogen Energy.

As we have seen, both $\Delta g_{r}^{\circ}$ and $\Delta h_{r}^{\circ}$ go to zero in sufficiently intense fields. Nevertheless, in decomposing water into hydrogen and oxygen, $\Delta h_{r 0}^{\circ}$ and $\Delta g_{r 0}^{\circ}$, must be supplied at some point. The question of where this occurs is one reason for pursuing the matter further.

It should be added that at field strength of $10^{4} \mathrm{~T}$ other magnetochemical effects may appear. The Brillouin function for molar magnetization may not be sufficient due to the appearance of spinorbital coupling and nuclear spin interaction. In addition, demagnetization from these fields may lead to microwave radiation. None of these effects have been evaluated.

\section{FIELD MAINTENANCE}

Energy is extracted from a magnetic field when oxygen is produced by thermomagnetic water decomposition. When oxygen is withdrawn from the field, magnetic energy is returned. These must be balanced to prevent either demagnetization of the magnet or the use of excessive external magnetic work. The electrical work of magnetization is given by (from eq. (2))

$$
\int B \mathrm{~d} m_{0}
$$

In Fig. 1 is shown a sketch of $m_{0}$ vs $B$ at various temperatures (the actual temperature effect has been exaggerated to better illustrate the point). If the reaction occurs isothermally (which would occur adiabatically when $\Delta h_{r}^{\circ}=0$ ), path $\mathrm{A}$ will be followed during the decomposition. If the oxygen is then removed adiabatically, it will undergo adiabatic demagnetization and follow path $B$. The area within the paths represents a net energy removed from the magnetic field. Conversely, if the reaction mixture is heated to a higher temperature during removal from the field, path $\mathrm{C}$ might be followed 


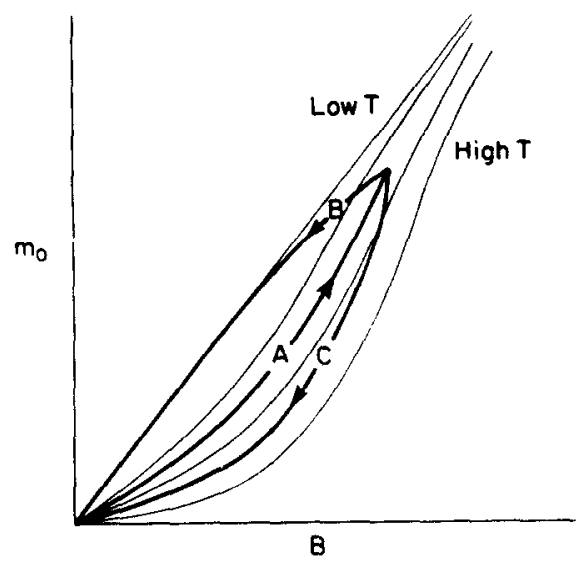

FIci. 1. Molar magnetization isotherms and alternate demagnetization paths.

and net magnetic energy will be given to the field (this could contribute to field maintenance in the face of coil losses). The temperature path during reaction and demagnetization must be carefully controlled. Isothermal reaction and demagnetization will be assumed for the purposes of further evaluation, and there will be no net energy exchange with the magnetic field.

To attain isothermal oxygen removal and demagnetization. thermal energy must be provided. This may be shown to be equal to $m_{\mathrm{o}} B$ per mole oxygen for isothermal removal from a field of strength $B$. From eq. (11) we see that

$$
\Delta h_{r}^{\circ}+\frac{1}{2} m_{0} B=\Delta h_{r 0}^{\circ},
$$

and therefore the total thermal energy that must be provided consists of the $\Lambda h_{r}$ during the reaction and $\frac{1}{2} m_{\mathrm{o}} B$ during isothermal demagnetization, for the total of $\Delta h_{\mathrm{r} 0}^{\circ}$, the original $(B=0)$ heat of reaction.

\section{THERMOMAGNETIC WATER-SPLITTING SYSTEM}

The system shown in Fig. 2 embodies the components of a direct thermomagnetic water-splitting scheme. Water vapor at relatively elevated temperature and pressure is provided by a boiler and fed

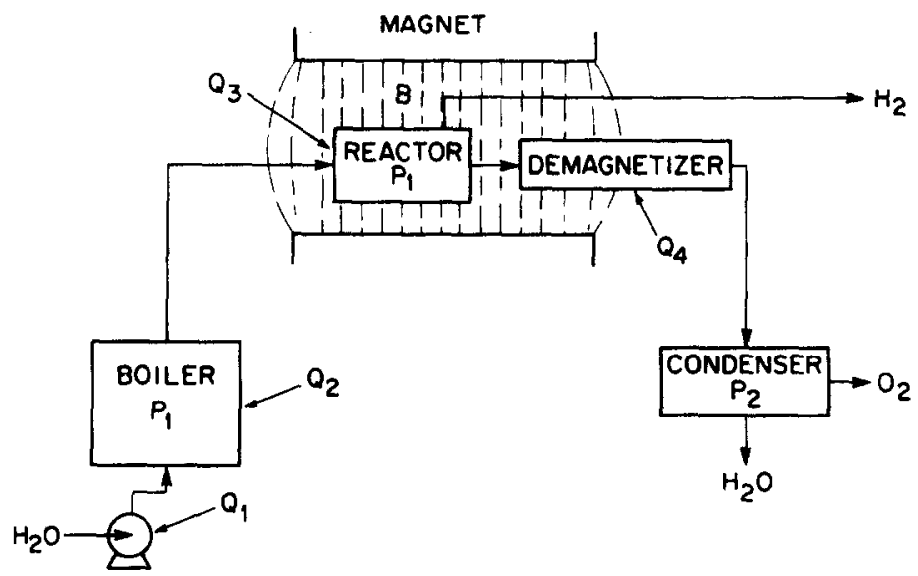

FIG. 2. Thermomagnetic water-splitting process. 
to a reactor contained between the poles of a magnet. The reactor is visualized as a tube-in-shell diffuser using palladium tubes through which $\mathrm{H}_{2}$. but not $\mathrm{O}_{2}$ or $\mathrm{H}_{2} \mathrm{O}$. may diffuse [13]. Hydrogen is removed from (say) the shell side of the reactor. while undecomposed water, oxygen and some residual $\mathrm{H}_{2}$ are removed at the end of the tubes. If a catalyst is required to enhance water decomposition it may be packed into the tubes.

It will be assumed for the purposes of a simple analysis that reaction occurs only within a region of uniform magnetic field and that none occurs in either the feed or product removal lines. Therefore the pressure in the reactor is that in the boiler, but this is significantly higher than in the condenser and $\mathrm{H}_{2} \mathrm{O} / \mathrm{O}_{2}$ separator because of the work required to remove a paramagnetic substance from a magnetic field. The hydrogen, being non-paramagnetic, is recovered at approximately the equilibrium pressure in the reactor (minus pressure drop necessary for diffusion through the palladium tubes which will be neglected here).

Assuming reversible isothermal expansion (for field maintenance) as the oxygen-rich stream leaves the field. the pressure change will be given by eq. (4). On the basis of one mole of water fed to the system, this may be expressed as

$$
\int_{P_{1}}^{P_{2}} \hat{v} \mathrm{~d} P=\int_{B}^{0} \hat{m} \mathrm{~d} B
$$

where $\hat{v}$ and $\hat{m}$ are the specific volume and specific magnetization on a common basis of one mole of water. If $X$ is the fractional conversion of the water fed and $\alpha$ is the fraction of $\mathbf{H}_{2}$ produced that is recovered in the $\mathrm{H}_{2}$ product stream $(\alpha<1.0)$. stoichiometry yields the following relations applicable to the oxygen-rich product stream (assuming ideal gas conditions)

$$
\hat{v}=[1+(0.5-x) X] \frac{R T}{P}
$$

and

$$
\hat{m}=\frac{1}{2} X m_{0}
$$

An assumption of no reaction between residual $\mathrm{H}_{2}$ and $\mathrm{O}_{2}$ in the oxygen product stream eliminates a rather complicated calculation for the expansion of this stream. Combining eqs (8) and (19), we obtain

$$
\frac{1}{X} \int_{P_{1}}^{r_{2}} \hat{\imath} \mathrm{d} P+\Delta g_{r}=\Delta g_{r 0}
$$

Since the first term is the flow-expansion work available per mole water reacted and $\Delta g_{r}^{\circ}$ is the (isothermal) work necessary to return the products to standard conditions (small for $K_{p}$ of the order of one), we see that the standard state $(B=0)$ work of water decomposition is being provided primarily by the pressure drop during demagnetization. The remainder $\left(\Delta g_{v}^{\circ}\right)$ is provided magnetically, but returned to the field during isothermal demagnetization.

Integrating eq. (22) using eqs (20) and (15) (for $B>10^{4} \mathrm{~T}$ ) yields

$$
\frac{(1+(0.5-\alpha) X)}{X} \ln \frac{P_{1}}{P_{2}}=\frac{2 N \beta B}{R T} \text {. }
$$

In addition, it follows from the definition of $K_{p}$ that

$$
K_{p}=\frac{P_{11} P_{O_{2}}^{1}{ }^{2}}{P_{\mathrm{H}_{1} \mathrm{O}}}=\frac{(1-\alpha) P_{1}^{1}{ }^{2} X^{32}}{(1-X)(2+X-2 \alpha X)^{1 / 2}}=K_{p 0} \exp \left(\frac{2 N \beta B}{R T}\right) .
$$

Equations (23) and (24) involve $P_{1}, P_{2}, B, \alpha, X$ and $T$, giving four degrees of freedom to the design decisions. We also perceive that $P_{1}$ and $T$ should be as high as possible, $B$ as low as possible, and $P_{2}$ not too small. compatible with a large $X$ and a large $\alpha$.

For the purposes of illustrating a design. I will choose the boiler to operate at $600 \mathrm{~K}$, without superheat, giving $P_{1}=121.6 \mathrm{~atm}$ (slightly below the critical point of water). In addition. assuming $\alpha-0.95$ (a "good" hydrogen recovery), we may obtain $X$ and $K_{p}$ (and $B$, and $P_{\mathrm{H}_{2}}$ ) in terms of $P_{2}$. The results are shown in Table 2.

We see that the lower the exhaust pressure $\left(P_{2}\right)$, the better the conversion and the higher the hydrogen pressure. This is because more flow work is available at the higher ratio of $P_{1} / P_{2}$. The field 
TABLE: 2. Alternative operating conditions for a thermomagnetic water-splitting system using $T=600 \mathrm{~K}, P_{1}=121.6 \mathrm{~atm}$ and $\alpha=0.95$

\begin{tabular}{lllll}
\hline $\begin{array}{c}P_{2} \\
\text { latm) }\end{array}$ & $X$ & $\begin{array}{c}P_{\mathrm{H}_{2}} \\
(\mathrm{~atm})\end{array}$ & $K$ & $\begin{array}{c}B \\
\text { Teslat }\end{array}$ \\
\hline 0.01 & 0.213 & 1.43 & 0.0511 & $1.786 \times 10^{4}$ \\
0.10 & 0.166 & 1.09 & 0.0329 & $1.766 \times 10^{4}$ \\
1.0 & 0.117 & 0.749 & 0.0181 & $1.740 \times 10^{4}$ \\
10 & 0.0639 & 0.400 & 0.00682 & $1.696 \times 10^{4}$ \\
100 & 0.90569 & 0.0347 & 0.000169 & $1.531 \times 10^{4}$ \\
\hline
\end{tabular}

intensity required is not strongly dependent upon $P_{2}$, but it should be observed that in this range $K_{p}$ is strongly dependent upon $B$.

Although water vapor at $600 \mathrm{~K}$ and $121.6 \mathrm{~atm}$ departs from the ideal gas law, the treatment here (using eq. (20)) is considered adequate for the present purposes.

It would be desirable to make the reactor and demagnetizer as small as possible in order to minimize the volume of high-intensity magnetic field. This will depend upon the area requirements for hydrogen diffusion and heat transfer.

\section{THERMAL EFFICIENCY}

It is apparent that with only minor irreversibilities present in the idealized thermomagnetic water splitting system, the thermal efficiency should approach the Carnot efficiency. Assuming that liquid water is provided at $25^{\circ} \mathrm{C}$ and that both hydrogen and oxygen are produced at $1 \mathrm{~atm}$ and $25^{\circ} \mathrm{C}$. we may calculate the heat requirements at each unit of the process. These are shown in Table 3 on the basis of 1 gmole water passing through the process with an exhaust pressure of $1 \mathrm{~atm}$ and a conversion of $X=0.117$. A $30 \%$ efficiency has been adopted for the conversion of heat to compression work.

The significant heat terms, that for the heating and vaporization of the water feed, and that for reaction and isothermal demagnetization. are comparable in magnitude. As has been seen, the former is providing the work required for water decomposition; the latter, the heat required.

TABLF 3. Heat requirements for the thermomagnetic water-splitting process, per gmole water thoughput (kJ)

\begin{tabular}{llc}
\hline$Q_{1}$ & Liquid water compression $(25 \mathrm{C})$ & 0.74 \\
$Q_{2}$ & Vaporization & 33.7 \\
$Q_{3}$ & Heat of reaction $\left(B=1.74 \times 10^{4} \mathrm{~T}: X=0.117\right)$ & 17.3 \\
$Q_{4}$ & Isothermal demagnetization & 11.4 \\
$Q_{5}$ & Hydrogen compression (to $1 \mathrm{~atm} .25^{\circ} \mathrm{Cl}$ & 0.27 \\
& & Total \\
\end{tabular}

The free energy of water decomposition at $25^{\circ} \mathrm{C}$ and $B=0$ is $228.5 \mathrm{~kJ} / \mathrm{gmole}$ and therefore the efficiency at $X=0.117$ and $\alpha=0.95$ is 0.40 . The comparative Carnot efficiency between 600 and $298 \mathrm{~K}$ is 0.50 . The departure from Carnot efficiency is due primarily to not recovering, in this simplified process description, the heat available in the $600 \mathrm{~K}$ oxygen rich product stream. Actual efficiencies will, of course, be somewhat lower, after fully accounting for heat exchange and other losses.

The greatest practical difficulty (apart from producing $B=1.74 \times 10^{4} \mathrm{~T}$ ) lies in the heat exchanger required for isothermal demagnetization. 


\section{SUMMARY}

It has been shown that water may be decomposed into hydrogen and oxygen by the use of at sufficiently strong magnetic field. Although the field required for a moderate conversion of water is far greater than any presently available. the problem reminds one somewhat of hydrogen-fusion power generation, where large volume intense magnetic fields up to $16.5 \mathrm{~T}$ are proposed $[10,14]$ and the working gases are heated to temperatures in the $\mathrm{keV}$ range. If the necessary intense fields could be obtained. a thermomagnetic water splitting process would be a "clean" system for providing future hydrogen without requiring a complex series of chemical reactions (in thermochemical methods) or temperatures in excess of about $600 \mathrm{~K}$.

Acknowledgement-Support and encouragement of this work was provided by Organization Control Services. Inc., of Ann Arbor, Michigan.

\section{REFERENCES}

1. E. A. Fletcher and R. L. MOFN. Sicience, N. Y. 197, 1050 (1977).

2. R. Delhez, Bull. Soc. R. Sci. liège 26, 83 (1957).

3. R. Delhez, Bull. Soc. R. Sci. Liẹgle 26, 161 (1957).

4. C.P. Chen, R. J. Heinsohn and L. N. Mulay, I. phys. Soc. Japan 25, 319 (1968).

5. E. A. Guggenheim, Thermodynamics (5th edn) Interscience, New York (1967).

6. J. S. Hsien, Principles of Thermodynamics. Scripta Book Co., Washington. D. C. (1975).

7. J. H. VAN Vieck. The Theory of Electric and Magnetic Susceptibilities. Oxford University Press. Oxford (1932). Dover Reprint (1966).

8. Physical and Thermodynumic Properties of Elements and Compounds. Chemetron Corp.. Louisville (1969).

9. Chem. Engng New's p. 18 (8 August 1977).

10. P. Komerek, Cyrogenics 16, 131 (1976).

11. D. H. Parkinson and B. E. Muliall, The Generation of High Magnetic Ficlds, Plenum Press, Niw York (1967)

12. J. Landstreet and J. R. P. Angel, Astrophys. I. 196, 918 (1975).

13. R. B. MCBride and D. L. MCKinley, Chem. Engng Prog. 61, 81 (1965).

14. G. A. CaRlson, Trans. Am. nucl. Soc. 26, 48 (June 1977). 